\title{
Electrostatic Torsion Mirror for Optical Disk Drives
}

\author{
Minoru Yonezawa, Masayuki Sekimura, Kiyotaka Uchimaru*, Norio Uchida, and Akihiro Kasahara \\ Mechanical Systems Research Laboratories, Toshiba Corp., 1, Komukai Toshiba-cho, Saiwai-ku, Kawasaki 210-8582, Japan \\ *Komukai Works, Toshiba Corp., 1, Komukai Toshiba-cho, Saiwai-ku, Kawasaki 210-8581, Japan
}

\begin{abstract}
A new electrostatic torsion mirror with a silicon/silicon structure has been developed for optical disk drives. It positions the beam spot accurately on an optical disk ${ }^{10,2)}$. This mirror is mounted on a $3.8 \times 4.5$ $\mathrm{mm}$ chip and weighs approximately $25 \mathrm{mg}$. Thus, downsizing of optical heads can be realized by using this torsion mirror. This torsion mirror is composed of an elliptic mirror plate, twin supporting beams, and a pair of driving electrodes. The forms of these elements define the mechanical parameters of this torsion mirror: natural frequency and damping ratio. These mechanical parameters are designed to satisfy the specifications for a high-frequency positioning of the optical beam spot. Experimental frequency responses showed that a test model had a second modal mechanical frequency of over $50 \mathrm{kHz}$, and the characteristics were in good agreement with the designed values. This torsion mirror will make possible more accurate spot positioning than can be achieved using conventional electromagnetic actuators.
\end{abstract}

Key words: torsion mirror, electrostatic force, positioning actuator, optical heads, silicon process, squeeze effect

\section{Introduction}

Optical disk memories have the potential to satisfy the growing need for large-capacity, high-speed and highdensity memory. In this regard, high-frequency positioning control is a key technology. For this control, positioning actuators with a high mechanical frequency are indispensable. These actuators, however, cannot be realized easily because of structural problems. For example, conventional electromagnetic actuators for optical disk drives have a maximum mechanical frequency of approximately $50 \mathrm{kHz}$, and have high gain peaks. We have developed a new electrostatic torsion mirror actuator that has a high mechanical frequency of over $50 \mathrm{kHz}$ and low gain peak.

This mirror actuator is fabricated as a compact chip using micro fabrication process. Recently this process has been applied to the fabrication of the fine positioning actuator for computer peripheral devices: HDD (Hard Disk Drives) and laser scanner ${ }^{37}$. In our application to the mirror actuator for optical disk drives, DRIE (Deep Reactive Ion Etching) is the key process to shape the mirror plate to the desired elliptic form and to shape deep grooves. The application of this process resulted in a compact and lightweight mirror actuator. By mounting this mirror actuator on an optical head, it is possible to realize a drastically downsized optical head. Simultaneously the ability for high-frequency positioning control can be obtained.

\section{Design}

\subsection{Specifications for electrostatic mirror actuator}

This mirror actuator finely positions the optical beam spot in the radial direction of an optical disk, in cooperation with a coarsely positioning actuator. Two schematic views of the optical spot positioning system are shown in Fig. 1. In this system, linear motor works as a coarsely positioning actuator, and the mirror actuator works as a finely positioning actuator. In the general positioning system, these two actuators position the beam spot to a tolerance of $\pm 5 \%$ of the track pitch against the repeatable disturbance caused by the disk motor. The coarse actuator mainly takes charge of disturbance reduction for frequency band of disk rotation, and the fine actuator takes charge of high-frequency settling to the target position and regulating the positioning error of approximately 10 tracks. This frequency shared control has realized broad frequency band control of over $5 \mathrm{kHz}$, which is required for narrow track pitch disks.

Here, on the assumption that a linear motor for this positioning system has mechanical frequency of less than $10 \mathrm{kHz}$, the mechanical parameters of the mirror actuator are restricted from the viewpoint of the control stability for the frequency sharing control.
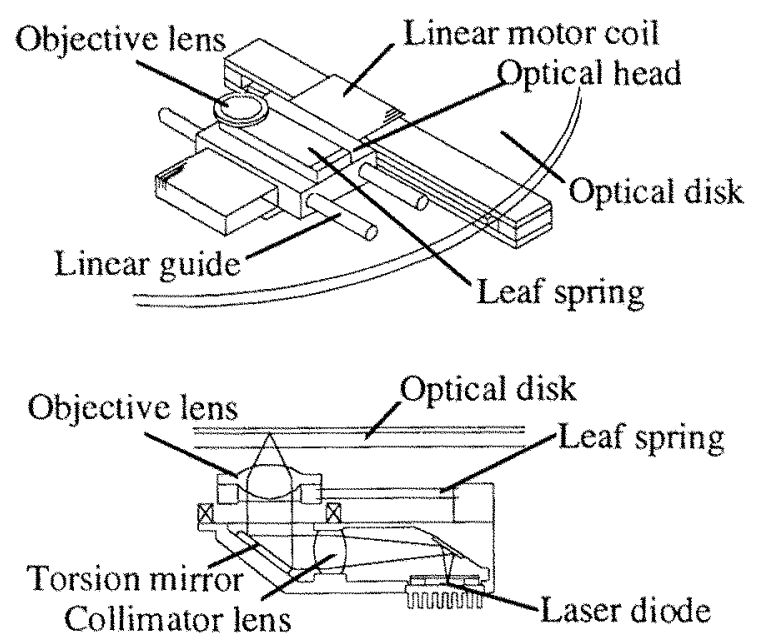

Fig. 1 Optical spot positioning system 


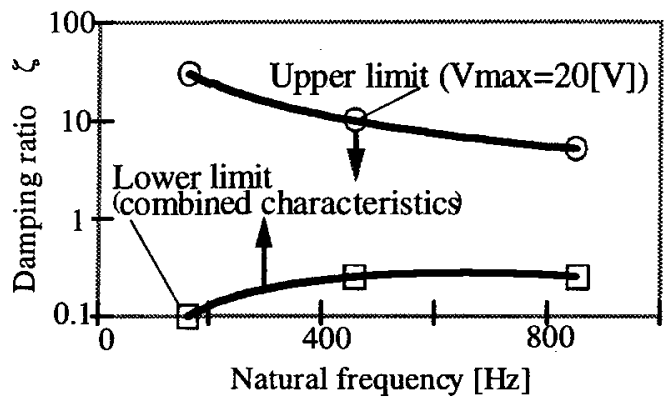

Fig. 2 Restraints for stable positioning control

Figure 2 shows the restraints on natural frequency and damping ratio of the fine actuator. The upper limit is calculated in accordance with the maximum input voltage and the lower limit is defined in accordance with the order of the positioning compensator.

\subsection{Dynamics of actuator}

The mirror actuator is composed of an elliptic mirror plate, twin supporting beams and a pair of driving electrodes (Fig. 3). The natural frequency of mirror rotation is defined by the moment of inertia of the mirror plate and the stiffness of the beams. The squeeze effect between the mirror plate and electrodes generates the damping force, and the grooves reduce the influence of the damping force. The equation of motion of the mirror actuator is as follows;

$$
I \ddot{\theta}+C \dot{\theta}+K \theta=M .
$$

Here, $I$ is the inertia moment, $C$ the air-damping coefficient, $K$ the torsional stiffness, $M$ the driving moment, and $\theta$ the rotation angle of the mirror plate. Natural frequency $f_{n}$ and damping ratio $\zeta$ are calculated using these parameters as follows;

$$
\begin{aligned}
& f_{n}=\sqrt{K / I} / 2 \pi \\
& \varsigma=C / 2 \sqrt{I K} .
\end{aligned}
$$

Based on these fundamental equations, mechanical parameters are designed.

Figure 4 shows the driving method of this mirror actuator. Inputting voltage to each electrode, the driving moment is generated as the electrostatic force. Here, this electrostatic force $f$ depends on the gap $d$ and input voltage $V$ as follows;

$$
f=\varepsilon S(V / d)^{2} / 2 \text {. }
$$

Here, $\varepsilon$ is the dielectric constant and $S$ the area of each electrode. Then Eq. (4) shows that this electrostatic force is nonlinear with the input voltage. Therefore, DC base voltage $V o$ is added to control variable $u$ in this torsion mirror operation as follows;

$$
\begin{aligned}
& V_{a}=V_{0}+u, V_{b}=V_{0}-u, \\
& M=M_{a}-M_{b} .
\end{aligned}
$$

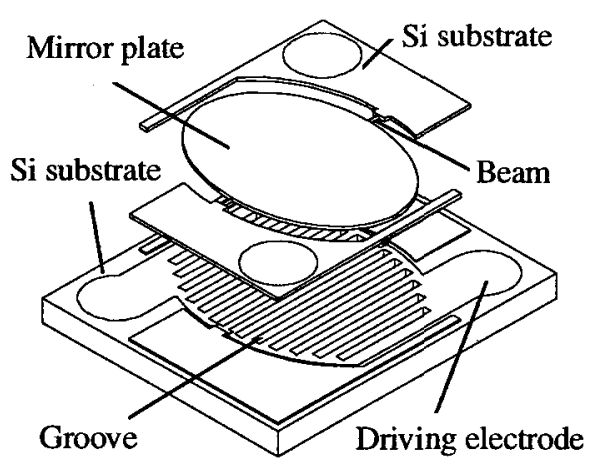

Fig. 3 Schematic view of electrostatic torsion mirror

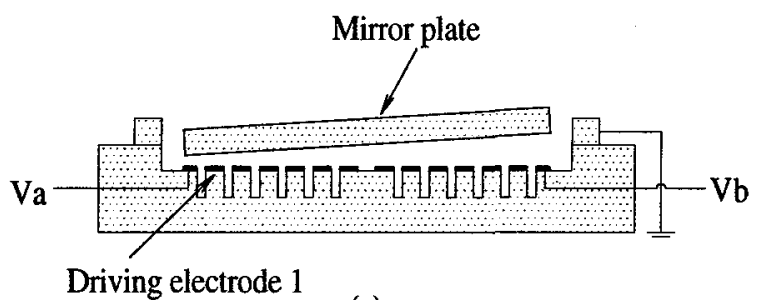

(a)

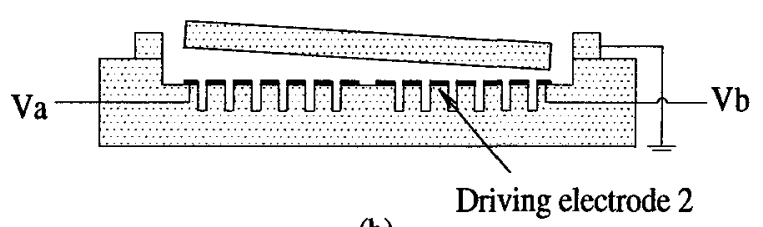

(b)

Fig. 4 Driving method

(a) $V a>V b$, (b) $V a<V b$

Here, $\mathrm{Va}$ is the voltage input in the one electrode, $V b$ the voltage input in the other electrode, $M a$ the driving moment generated in the one electrode, $M b$ the driving moment generated in the other electrode. With this driving method, the driving moment is generated keeping the linearity with the control variable in the condition that the rotation angle is small.

\subsection{Design of the mechanical parameters}

A mirror actuator has been designed, whose mechanical parameters satisfy the restraints for control stability considering the dynamics of the actuator. The mechanical parameters of supporting beams are designed, so that mechanical frequency is set as $1000 \mathrm{~Hz}$. The mechanical parameters of grooves are designed by damping force calculation using Reynolds equation analysis, so that the damping ratio satisfies the restraints.

The dimensions of the mirror actuator chip are $3.8 \mathrm{x}$ $4.5 \mathrm{~mm}$. The mirror plate is designed in an elliptic shape to reflect $2 \mathrm{~mm}$ diameter incident light beam, and the supporting beams are $11 \mu \mathrm{m}$ wide, $60 \mu \mathrm{m}$ high and $200 \mu \mathrm{m}$ long. The grooves are $120 \mu \mathrm{m}$ wide and $100 \mu \mathrm{m}$ deep. Other specifications are shown in Table 1. 
Table 1 Specifications for electrostatic torsion mirror

\begin{tabular}{|c|r|}
\hline Major axis length of mirror & $3110 \times 10^{6}[\mathrm{~m}]$ \\
\hline Minor axis length of mirror & $2200 \times 10^{-6}[\mathrm{~m}]$ \\
\hline Width of a beam & $11 \times 10^{-6}[\mathrm{~m}]$ \\
\hline Length of a beam & $200 \times 10^{-6}[\mathrm{~m}]$ \\
\hline Thickness of mirror plate & $60 \times 10^{-6}[\mathrm{~m}]$ \\
\hline Gap between mirror and electrodes & $7 \times 10^{-6}[\mathrm{~m}]$ \\
\hline Groove width & $120 \times 10^{-6}[\mathrm{~m}]$ \\
\hline Groove depth & $100 \times 10^{-6}[\mathrm{~m}]$ \\
\hline
\end{tabular}

\section{Fabrication}

The mirror actuator is fabricated using two substrates of (100) silicon wafer. These two substrates are bonded by a water glass bonding method ${ }^{4}$. Supporting beams and mirror plate in the upper substrate and deep grooves in the lower base substrate are fabricated by DRIE. The process sequence of fabrication is shown in Fig. 5 .

a) Double side polished, (100) silicon wafer of $500 \mu \mathrm{m}$ thickness for low base substrate is oxidized.

b) Silicon dioxide is patterned to fabricate a gap on the front side and alignment mark on the back side. Subsequently, the wafer is anisotropically etched 7.45 $\mu m$ using tetramethyl ammonium hydroxide (TMAH) solution.

c) After silicon dioxide is stripped, the wafer is oxided again. Subsequently, $3000 \AA \mathrm{Au} / 1500 \AA \mathrm{Cr}$ for driving electrodes is deposited and patterned.

d) Silicon dioxide is patterned for grooves. Subsequently, the wafer is etched $100 \mu \mathrm{m}$ by DRIE.

e) Double side polished, (100) wafer of $60 \mu \mathrm{m}$ thickness for an upper substrate is oxidized.

f) The upper silicon wafer and the lower base on wafer are bonded with water glass.

g) Silicon dioxide on the upper silicon wafer is patterned to fabricate supporting beams and mirror plate. Subsequently, the upper silicon wafer is etched by DRIE.

\section{Results}

A photograph of the mirror actuator is shown in Fig. 6. This photograph clarifies that the mirror actuator has a desired elliptic mirror plate, which is supported by twin beams. Furthermore, the grooves are proved to have the designed form.

Figure 7 shows the experimental results of frequency characteristics measured by inputting control voltage to the driving electrodes in the condition of $\mathrm{Vo}=5[\mathrm{~V}]$. The control variable is less than $5[\mathrm{~V}]$, and thus the mirror plate rotates less than $\pm 0.5 \mathrm{mrad}$. According to the experimental frequency responses, the mirror actuator is certified to have a high mechanical frequency of over 50
$\mathrm{kHz}$ and low gain peaks. Furthermore, the experimental mechanical parameters are identified using these results (Fig. 8), and proved to be in good agreement with the designed values. Table 2 shows the comparison between designed and identified values.

\section{Conclusions}

The restricted mechanical relationship between natural frequency and damping ratio of a mirror actuator is clarified, which must be satisfied to make the positioning control stable. And a mirror actuator is designed, whose mechanical parameters satisfy the restraints. The actuator is fabricated by DRIE. It has been confirmed that the actuator has the designed shape and mechanical parameters. Furthermore, this actuator is experimentally certified to have a high mechanical frequency, and thus high-frequency positioning control can be realized using this actuator.

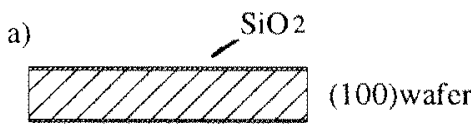

b)

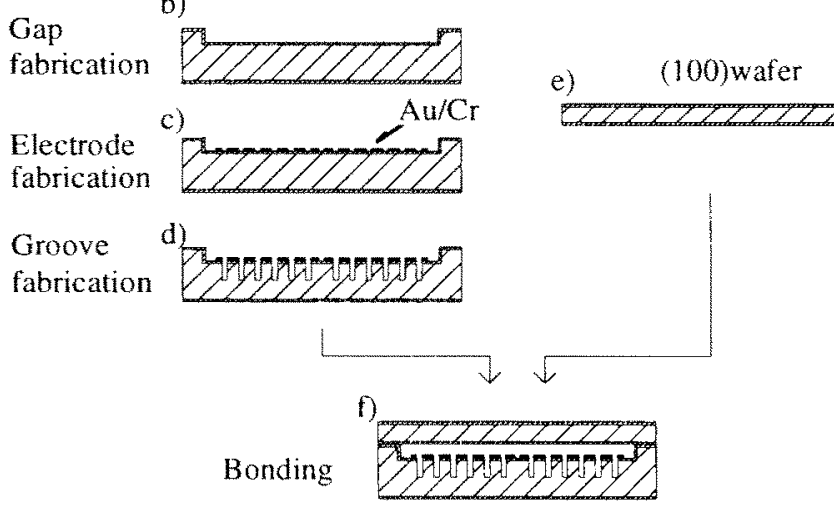

g)

Mirror \& beams fabrication

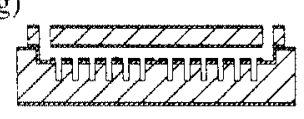

Fig. 5 Fabrication process

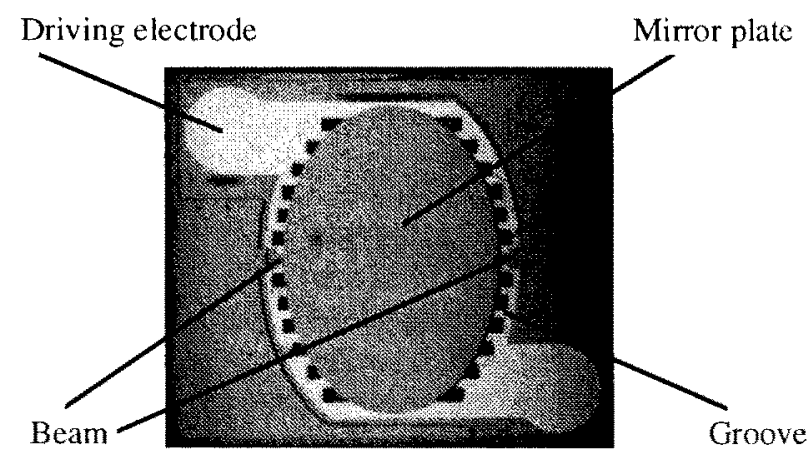

Fig. 6 Photograph of test model 

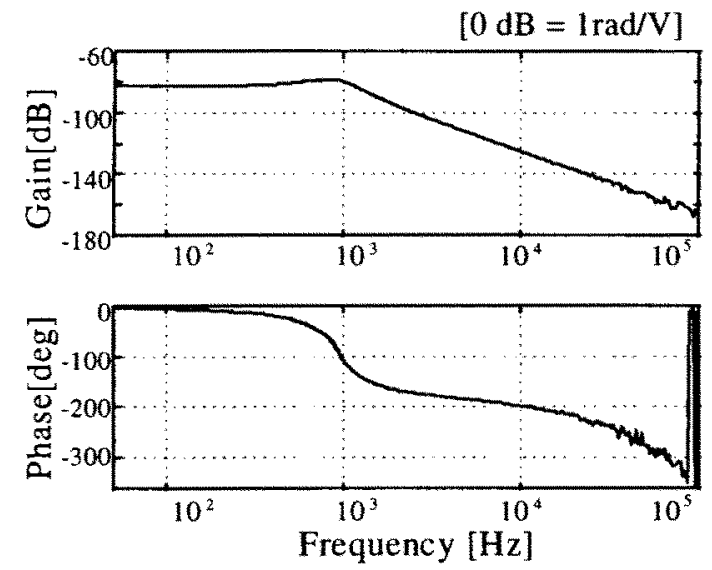

Fig. 7 Experimental frequency characteristics of mirror actuator $(\mathrm{Vo}=5[\mathrm{~V}])$
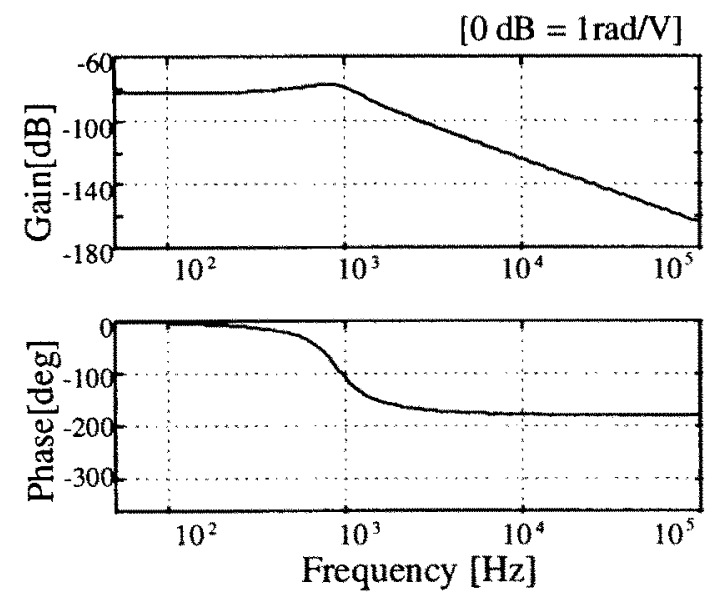

Fig. 8 Identified frequency characteristics of mirror actuator $\left(\mathrm{Vo}_{\mathrm{O}}=5[\mathrm{~V}]\right)$
Table 2 Identified parameters of the test model $(\mathrm{Vo}=5[\mathrm{~V}])$

\begin{tabular}{|c|c|c|}
\hline DC gain & $\begin{array}{c}\text { Designed } \\
1.1 \times 10^{-4} \\
{[\mathrm{rad} / \mathrm{V}]}\end{array}$ & $\begin{array}{c}\text { Identified } \\
0.8 \times 10^{-4} \\
{[\mathrm{rad} / \mathrm{V}]}\end{array}$ \\
\hline Natural frequency & $1000[\mathrm{~Hz}]$ & $900[\mathrm{~Hz}]$ \\
\hline Damping ratio & 0.44 & 0.6 \\
\hline
\end{tabular}

\section{References}

1) M.Sekimura, M.Yonezawa, K. Uchimaru, A. Kasahara and N. Uchida : 'Electrostatic Torsion Mirror', Tech. Dig. of $16^{\text {th }}$ Sensor Symposium, 1998, pp.167-170

2) M.Yonezawa, M.Sekimura, K. Uchimaru, N. Uchida, T. Sugaya and A. Kasahara : 'Electrostatic Torsion Mirror for Optical Heads', J. ITE Jpn., 52, pp.1507-1512 (1998)

3) H.Fujita and K.J.Gabriel : 'New Opportunities for Micro Actuators', Dig. of Technical Papers, Transducer '91, pp.1420 (1991)

4) H.J.Quenzer, W.Beneche and C. Dell: 'Low Temperature Water Bonding for Micromechanical Application', Micro Electro Mechanical Systems 92 (MEMS '92), Travemunde (Germany), February 4-7, 1992 